\title{
Biologically effective dose and prediction of obliteration of unruptured arteriovenous malformations treated by upfront Gamma Knife radiosurgery: a series of 149 consecutive cases
}

\author{
*Constantin Tuleasca, MD, PhD, ${ }^{1-4}$ Iulia Peciu-Florianu, MD, ${ }^{1}$ Henri-Arthur Leroy, MD, ${ }^{1}$ \\ Maximilien Vermandel, PhD, ${ }^{1,6}$ Mohamed Faouzi, PhD, ${ }^{5}$ and Nicolas Reyns, MD, PhD ${ }^{1,6}$ \\ ${ }^{1}$ Centre Hospitalier Regional Universitaire de Lille, Roger Salengro Hospital, Neurosurgery and Neurooncology Service, Lille, \\ France; 'Lausanne University Hospital (CHUV), Department of Clinical Neurosciences, Neurosurgery Service and Gamma Knife \\ Center, Lausanne; ${ }^{3}$ University of Lausanne, Faculty of Biology and Medicine, Lausanne; ${ }^{2}$ Signal Processing Laboratory (LTS \\ 5), Ecole Polytechnique Fédérale de Lausanne; ${ }^{5}$ Division of Biostatistics, Center for Primary Care and Public Health (Unisanté), \\ University of Lausanne, Switzerland; and 'University of Lille, Inserm, CHU Lille, U1189-ONCO-THAI-Image Assisted Laser \\ Therapy for Oncology, Lille, France
}

OBJECTIVE Arteriovenous malformations (AVMs) present no pathologic tissue, and radiation dose is confined in a clear targeted volume. The authors retrospectively evaluated the role of the biologically effective dose (BED) after Gamma Knife radiosurgery (GKRS) for brain AVMs.

METHODS A total of 149 consecutive cases of unruptured AVMs treated by upfront GKRS in Lille University Hospital, France, were included. The mean length of follow-up was 52.9 months (median 48, range 12-154 months). The primary outcome was obliteration, and the secondary outcome was complication appearance. The marginal dose was $24 \mathrm{~Gy}$ in a vast majority of cases ( $n=115,77.2 \%$; range $18-25 \mathrm{~Gy}$ ). The mean BED was $220.1 \mathrm{~Gy}_{2.47}$ (median 229.9, range 106.7-246.8 $\mathrm{Gy}_{2.47}$ ). The mean beam-on time was 32.3 minutes (median 30.8, range 9-138.7 minutes). In the present series, the mean radiation dose rate was $2.259 \mathrm{~Gy} / \mathrm{min}$ (median 2.176, range 1.313-3.665 Gy/min). The Virginia score was 0 in 29 (19.5\%), 1 in 61 (40.9\%), 2 in 41 (27.5\%), 3 in 18 (12.1\%), and 4 in 0 (0\%) patients, respectively. The mean Pollock-Flickinger score was 1.11 (median 1.52, range 0.4-2.9). Univariate (for obliteration and complication appearance) and multivariate (for obliteration only) analyses were performed.

RESULTS A total of 104 AVMs (69.8\%) were obliterated at the last follow-up. The strongest predictor for obliteration was BED $(p=0.03)$. A radiosurgical obliteration score is proposed, derived from a fitted multivariable model: $(0.018 \times$ $\mathrm{BED})+(1.58 \times \mathrm{V} 12)+(-0.013689 \times$ beam-on time $)+(0.021 \times$ age $)-4.38$. The area under the receiver operating characteristic curve was 0.7438 ; after internal validation using bootstrap methods, it was 0.7088 . No statistically significant relationship between radiation dose rate and obliteration was found $(p=0.29)$. Twenty-eight $(18.8 \%)$ patients developed complications after GKRS; 20 (13.4\%) of these patients had transient adverse radiological effects (perilesional edema developed). Predictors for complication appearance were higher prescription isodose volume $(p=0.005)$ and $12-G y$ isodose line volume (V12; $p=0.001)$, higher Pollock-Flickinger $(p=0.02)$ and Virginia scores $(p=0.003)$, and lower beam-on time $(p=0.03)$.

CONCLUSIONS The BED was the strongest predictor of obliteration of unruptured AVMs after upfront GKRS. A radiosurgical score comprising the BED is proposed. The V12 appears as a predictor for both efficacy and toxicity. Beam-on time was illustrated as statistically significant for both obliteration and complication appearance. The radiation dose rate did not influence obliteration in the current analysis. The exact BED threshold remains to be established by further studies.

https://thejns.org/doi/abs/10.3171/2020.4.JNS201250

KEYWORDS stereotactic radiosurgery; Gamma Knife; biologically effective dose; obliteration; score; vascular disorders

ABBREVIATIONS AUC = area under the ROC curve; AVM = arteriovenous malformation; BED = biologically effective dose; DSA = digital subtraction angiography; GKRS = Gamma Knife radiosurgery; PIV = prescription isodose volume; RDR = radiation dose rate; ROC = receiver operating characteristic; SRS = stereotactic radiosurgery; V12 = 12-Gy isodose line volume.

SUBMITTED April 13, 2020. ACCEPTED April 24, 2020.

INCLUDE WHEN CITING Published online July 24, 2020; DOI: 10.3171/2020.4.JNS201250.

* M.F. and N.R. contributed equally to this work and share senior authorship. 
A RTERIOVENOUS malformations (AVMs) of the brain are considered congenital abnormalities of the blood vessels that derive from capillary networks, further permitting direct connections between cerebral arteries and veins. ${ }^{1,2}$ The most common symptoms are cerebral hemorrhage and seizures. ${ }^{3}$ Of note, annual hemorrhage rates are approximately $2 \%-4 \%$ for all brain AVMs and only $1 \%-3 \%$ for unruptured AVMs. ${ }^{4}$ Thus, it has been commonly considered that the natural history of unruptured AVMs is more benign than that of ruptured AVMs. ${ }^{5}$ Therapeutic options include microsurgical resection, ${ }^{6-8}$ endovascular treatment, ${ }^{9}$ and stereotactic radiosurgery $(\mathrm{SRS})^{10,11}$ used alone or in the frame of a multidisciplinary approach. ${ }^{12-14}$

In the present study, we investigated the role of the biologically effective dose (BED) on the outcomes after upfront SRS by Gamma Knife radiosurgery (GKRS) for unruptured AVMs. Of note, BED reflects the biological effectiveness of the total physical dose applied during a certain time. ${ }^{15}$ Unlike the radiation dose, BED integrates a time factor correction, accounting for DNA repair. Previously, the particular role of BED in single-fraction SRS treatments was rarely evaluated and was proposed for tumors, such as vestibular schwannomas, ${ }^{15}$ or in functional indications, such as trigeminal neuralgia. ${ }^{16}$ In particular, and of interest, in AVMs there is no pathological tissue, and the delivered dose is focalized on a confined target volume (unlike in some of the functional indications, in which the dose is prescribed to one point, like in trigeminal neuralgia). Moreover, it is now well established that SRS histologically engenders degeneration and proliferation of the vessel wall and conjunctive tissue changes, with further coagulopathy and respective AVM obliteration. ${ }^{17}$ In order to evaluate a radiobiological model as straightforwardly as possible, patients with previous hemorrhage (subject to different physiopathology and eventual radiobiology) ${ }^{18}$ and previous treatment modalities (reputed to have different results) were excluded..$^{19}$

\section{Methods \\ Study Design}

The design of this study was retrospective and nonrandomized. All patients gave written informed consent. The ethics committee of Lille University Hospital (CHU Roger Salengro) provided formal approval. All patients were consecutive cases treated in our center (Lille, France).

\section{Patient Population}

The indication for GKRS is discussed either in a multidisciplinary staff for cases coming from our hospital or for those in the neighboring areas, or with the referral physician for cases outside our hospital from all over France.

The inclusion criteria were patients older than 18 years at the time of treatment, diagnosed with nonhemorrhagic single AVMs, and solely treated by GKRS, with a minimum follow-up of 3 years. We excluded patients with prior hemorrhage, prior or further treatment independent of the modality, associated vascular abnormalities (e.g., cavernous malformations, dural arteriovenous fistulas, and multiple AVMs), or less than 3 years of follow-up. As an addi- tional clarifying point, during this period, we did not treat any unruptured AVMs in pediatric patients. In our unit, a vast majority of the pediatric cases are ruptured AVMs at the moment of treatment. Part of Lille's experience in pediatric AVM was previously published (using a linear accelerator) by the senior author of the current paper. ${ }^{20}$

We initially identified for further on-file analysis 172 consecutive patients with unruptured brain AVMs treated by GKRS at our university hospital over a period of more than 10 years, between April 2004 and September 2013. Twenty-three patients underwent a second GKRS and were excluded. We finally focused on 149 cases treated using upfront GKRS for unruptured AVMs. No patient underwent embolization or staged-volume radiosurgery, and no patient was lost to follow-up.

The demographic data are listed in Table 1 . The mean follow-up period was 52.9 months (median 48, range 12-154 months). The mean patient age was 40.5 years (median 40, range 18-68 years). The initial symptom was epilepsy in $66(44.3 \%)$, headaches in 34 (22.8\%), incidental in $32(21.5 \%)$, and neurological signs in 17 (11.4\%) patients. The AVM location was lobar supratentorial in 128 $(85.9 \%)$, infratentorial in $8(5.4 \%)$, thalamus in $6(4 \%)$, ventricular in $6(4 \%)$, and brainstem in $1(0.7 \%)$ patient. The AVM was located in eloquent areas in $95(63.8 \%)$ patients. The venous drainage was superficial in a vast majority of cases $(117,78.5 \%)$. There was an associated intranidal aneurysm in 10 patients $(6.7 \%)$. The Spetzler-Martin grade was I in $42(28.2 \%)$, II in 71 (47.7\%), III in 36 (24.2\%), and IV in $0(0 \%)$ patients. Cases with Spetzler-Martin grade III, and especially grades IV and V, usually had a higher volume and previously benefitted from embolization or were treated by staged GKRS, explaining their absence from the present cohort (see previous paragraph for case description).

\section{BED Calculation}

The BED was calculated using the simplified formula of Jones and Hopewell, ${ }^{21}$ or the basic BED model, as described by other authors, taking into account the irradiation time and the prescribed dose in the frame of a monoexponential fit equation. A methodology similar to the one we applied in this study was recently used by Graffeo et al. to evaluate the effect of single-fraction SRS for acromegaly in biological remission, and further analyzed in a short comment by Régis et al. ${ }^{41,42}$

The mean BED in the present series was $220.1 \mathrm{~Gy}_{2.47}$ (median 229.9, range 106.7-246.8 $\mathrm{Gy}_{2.47}$ ). Of note, although a vast majority of patients (almost $80 \%$ ) received a constant marginal dose of $24 \mathrm{~Gy}$, the BED, as adjusted by the treatment time (commonly associated with beam-on time for GKRS devices), varied significantly. By convention and to differentiate it from the measurement unit currently used for marginal dose (Gy), the measurement unit for $\mathrm{BED}$ was considered $\mathrm{Gy}_{2.47}$, as in a previous article by Tuleasca et al. on a related topic. ${ }^{16}$

\section{Beam-On Time and Radiation Dose Rate}

The mean beam-on time was 32.3 minutes (median 30.8 , range $9-138.7$ minutes). The mean radiation dose 
TABLE 1. Basic demographic data

\begin{tabular}{|c|c|}
\hline Variable & Value \\
\hline No. of patients & 149 \\
\hline \multicolumn{2}{|l|}{ Age, yrs } \\
\hline Mean & 40.5 \\
\hline Median (range) & $40(18-68)$ \\
\hline \multicolumn{2}{|l|}{ Sex } \\
\hline Male & $67(45)$ \\
\hline Female & $82(55)$ \\
\hline \multicolumn{2}{|l|}{ Common presenting symptoms } \\
\hline Epilepsy & $66(44.3)$ \\
\hline Headaches & $34(22.8)$ \\
\hline Incidental & $32(21.5)$ \\
\hline Neurological signs & $17(11.4)$ \\
\hline Prior AVM embolization & $0(0)$ \\
\hline Prior AVM resection & $0(0)$ \\
\hline Prior AVM irradiation treatments & $0(0)$ \\
\hline \multicolumn{2}{|l|}{ AVM location } \\
\hline Supratentorial lobar & $128(85.9)$ \\
\hline Infratentorial & $8(5.4)$ \\
\hline Thalamus & $6(4)$ \\
\hline Ventricular & $6(4)$ \\
\hline Brainstem & $1(0.7)$ \\
\hline Eloquent area & $95(63.8)$ \\
\hline \multicolumn{2}{|l|}{ Venous drainage } \\
\hline Superficial & $117(78.5)$ \\
\hline Deep & $32(21.5)$ \\
\hline Associated aneurysm & $10(6.7)$ \\
\hline \multicolumn{2}{|l|}{ Spetzler-Martin grade } \\
\hline I & $42(28.2)$ \\
\hline$\|$ & $71(47.7)$ \\
\hline III & $36(24.2)$ \\
\hline IV & $0(0)$ \\
\hline \multicolumn{2}{|l|}{ Follow-up duration, mos } \\
\hline Mean & 52.9 \\
\hline Median (range) & $48(12-154)$ \\
\hline
\end{tabular}

Values represent the number of patients (\%) unless stated otherwise.

rate (RDR) was $2.259 \mathrm{~Gy} / \mathrm{min}$ (median 2.176, range 1.313$3.665 \mathrm{~Gy} / \mathrm{min}$ ). The calibration dates were July 2003, with an RDR of $3.744 \mathrm{~Gy} / \mathrm{min}$, and April 2011, with an RDR of $3.212 \mathrm{~Gy} / \mathrm{min}$. The first GKRS treatment for other indication was performed in Lille in January 2004.

\section{GKRS Technique}

All patients underwent single-fraction SRS by GKRS with a Leksell Gamma Knife 4C model (Elekta Instruments, AB) using the Leksell GammaPlan. We always apply the Leksell stereotactic $\mathrm{G}$ frame under local anesthesia. All patients underwent stereotactic MRI and digital subtraction angiography (DSA).

Our philosophy is similar to that of the Pittsburgh group, ${ }^{22,23}$ as we do not routinely contour the AVM's nidus on multimodal imaging. Moreover, we report the equivalent of the prescription isodose volume (PIV) at the mo- ment of targeting. We usually prescribe a standard dose of $24 \mathrm{~Gy}$, which can be further modulated by the potentially critical anatomical location (e.g., brainstem [usually 12 Gy at the brainstem marginal contact], optic pathways [8 Gy maximal dose], tectal plate [no clear dose limit in the literature, considered sensitive to radiation by our group and treated with particular care]), and PIV, as well as the corresponding volume received by the 12-Gy isodose line.

No GKRS treatment was interrupted during irradiation delivery.

Dosimetric data can be seen in Table 2. The mean corresponding PIV was $2.44 \mathrm{~cm}^{3}$ (median 2, range 0.09-10 $\mathrm{cm}^{3}$ ). The mean volume corresponding to the 12-Gy isodose line (V12) was $6.7 \mathrm{~cm}^{3}$ (median 5, range 0.2-34.4 $\left.\mathrm{cm}^{3}\right)$. The marginal dose was $18 \mathrm{~Gy}$ in 2 patients (1.3\%), 20 Gy in 2 patients (1.3\%), 21 Gy in 20 patients (13.4\%), 22 Gy in 1 patient $(0.7 \%), 23$ Gy in 1 patient $(0.7 \%), 24 \mathrm{~Gy}$ in 115 patients $(77.2 \%)$, and 25 Gy in 8 patients (5.4\%), respectively. The Virginia score ${ }^{24}$ was 0 in $29(19.5 \%), 1$ in 61 (40.9\%), 2 in 41 (27.5\%), 3 in 18 (12.1\%), 4 in $0(0 \%)$ patients. The mean Pollock-Flickinger score ${ }^{25}$ was 1.11 (median 1.52, range 0.4-2.9).

\section{Primary and Secondary Outcomes}

The primary outcome was obliteration, and the secondary outcome was complication appearance.

\section{Follow-Up}

We routinely evaluate patients both clinically and radiologically at the 12-, 24-, 36-, 48-, and 60-month followup visits. Initially, brain MRI is acquired. When the AVM is considered completely obliterated, DSA is performed as a gold standard for confirming this outcome. In the present cohort, in $80 \%$ of cases DSA confirmed the obliteration, while MRI confirmed the remaining 20\%. Our group has also developed, over time, special sequences to further evaluate AVM obliteration by MRI, particularly for smallsized ones. ${ }^{26}$

\section{Statistical Analysis}

Statistical analysis was performed using Stata 14 (StataCorp). Descriptive statistics are reported as proportion or frequency for categorical data and mean, median, and range for continuous variables. Significance was set at $\mathrm{p} \leq 0.05$.

Obliteration and complication are both two binary outcomes. To assess the association between each predictor and the outcome, we performed a univariate logistic regression analysis. The strength and the significance of the association were measured using the odds ratio and the calculated $\mathrm{p}$ value. Fractional polynomial analysis was used to check for the functional relationship between each continuous predictor and the outcome. The linearity assumption was confirmed for the age, the BED, the V12, and the beam-on time, but not for the other variables. Additionally, for obliteration after GKRS, survival over time was examined using the Kaplan-Meier method (Fig. 1A). Patient censoring occurred at the moment of obliteration or at last follow-up. 
TABLE 2. Basic dosimetric data

\begin{tabular}{|c|c|c|c|c|c|}
\hline Variable & Mean & SD & Median & Range & No. of Patients (\%) \\
\hline BED & 220.1 & 25 & 229.9 & $106.7-246.8$ & \\
\hline Beam-on time, mins & 32.3 & 18.1 & 30.8 & $9-138.7$ & \\
\hline Vol corresponding to PIV, $\mathrm{cm}^{3}$ & 2.44 & 2.1 & 2 & $0.09-10$ & \\
\hline Vol of the $12 \mathrm{~Gy}, \mathrm{~cm}^{3}$ & 6.7 & 6.2 & 5 & $0.2-34.4$ & \\
\hline Dose at $50 \%$ isodose line, Gy & 23.5 & 1.3 & 24 & $18-25$ & \\
\hline 18 & & & & & $2(1.3)$ \\
\hline 20 & & & & & $2(1.3)$ \\
\hline 21 & & & & & $20(13.4)$ \\
\hline 22 & & & & & $1(0.7)$ \\
\hline 23 & & & & & $1(0.7)$ \\
\hline 24 & & & & & $115(77.2)$ \\
\hline 25 & & & & & $8(5.4)$ \\
\hline Virginia radiosurgery AVM score & 1.32 & 0.9 & 1.5 & $0-3$ & \\
\hline 0 & & & & & $29(19.5)$ \\
\hline 1 & & & & & $61(40.9)$ \\
\hline 2 & & & & & $41(27.5)$ \\
\hline 3 & & & & & $18(12.1)$ \\
\hline 4 & & & & & $0(0)$ \\
\hline Pollock-Flickinger score & 1.11 & 0.4 & 1.52 & $0.4-2.9$ & \\
\hline Delay of obliteration, mos & 45.5 & 12.7 & 36 & $12-96$ & 104 (69.8) obliterated \\
\hline
\end{tabular}

Given the insufficient number of complications, no multivariate analysis was performed for this outcome. However, for the obliteration, potential interactions were tested, and a multivariable model that optimizes the discrimination power between obliterated and not-obliterated AVMs was fitted. To overcome the violation of the linearity assumption for the V12 predictor (Fig. 2), we coded it into two categories ( 0 when the V12 was within $1-3.5 \mathrm{~cm}^{3}$ and 1 when the V12 was not within $\left.1-3.5 \mathrm{~cm}^{3}\right)$. Standard diagnostic model routines for the logistic model and the Hosmer-Lemeshow goodness-of-fit test were performed to assess the quality of the model fit and calibration. The area under the receiver operating characteristic (ROC) curve (AUC) and its $95 \%$ confidence interval were calculated to assess the discriminative performance of the final model. Finally, an obliteration score was built as the sum of the factors retained in the multivariable model, weighted according to their $\beta$-coefficients $=\log (\mathrm{OR})$.

\section{Internal Validation of the Score}

Harrell et al. ${ }^{27}$ discussed the principal methods (datasplitting, cross-validation, and bootstrapping) for obtaining nearly unbiased internal assessments of accuracy. However, it has been shown that cross-validation and data-splitting methods are relatively inefficient and need a large sample because a part of the data set is used for model development and the other part for the test. Given our relatively small sample, we preferred the bootstrapping method, which has the advantage that the entire data set is used for model development and provides nearly unbiased estimates of predictive accuracy. We performed the bootstrap method (repeated 1000 times) as described by Harrell et al. ${ }^{27}$ The algorithm allows calculating a virtually unbiased estimate of the external predictive discrimi- nation as the difference between the AUC (original model) and the optimism estimated from the bootstrap procedure.

\section{Results \\ Obliteration}

A total of $104(69.8 \%)$ AVMs were obliterated at last follow-up, after a median duration of 36 months (range 12-96 months).

The actuarial probabilities of obliteration at 24 (number at risk 142), 36 (number at risk 113), 48 (number at risk 78), 60 (number at risk 51), 72 (number at risk 41), and 96 months (number at risk 13) were 12.1\%, 37.9\%, 55.6\%, $63.3 \%, 72 \%$, and $76 \%$, respectively (Fig. 1A). Univariate logistic regression analysis showed no statistical significance for age $(\mathrm{p}=0.32)$, dose $(\mathrm{p}=0.10)$, Pollock-Flickinger score $(\mathrm{p}=0.68)$, or other variables (Table 3$)$. The relationship between BED and treatment time is shown in Fig. 1B, while the vast majority of patients received $24 \mathrm{~Gy}$. Moreover, there was a linear relationship between BED and obliteration (Fig. 1C) compared with the one corresponding to the dose (Fig. 1D). The AUC for BED and obliteration was 0.609 , while that for dose and obliteration was 0.56 (Fig. 1E and F). The multivariate model shows that obliteration was significantly related to beam-on time $(\mathrm{p}=0.05 ;$ OR 0.981 [SE 0.009], 95\% CI 0.962-1.001) and BED, which was the strongest predictor $(\mathrm{p}=0.03$; OR 1.015 [SE 0.007], 95\% CI 1.001-1.029). These predictors taken together show a fairly good predictive power of obliteration with an $\mathrm{AUC}=0.7438$.

No statistically significant relationship between RDR and obliteration was found (OR 1.33 [SE 0.363], 95\% CI $0.78-2.27, \mathrm{p}=0.29$ ). Interestingly, the AUC was 0.56, identical to that found for dose and obliteration. 

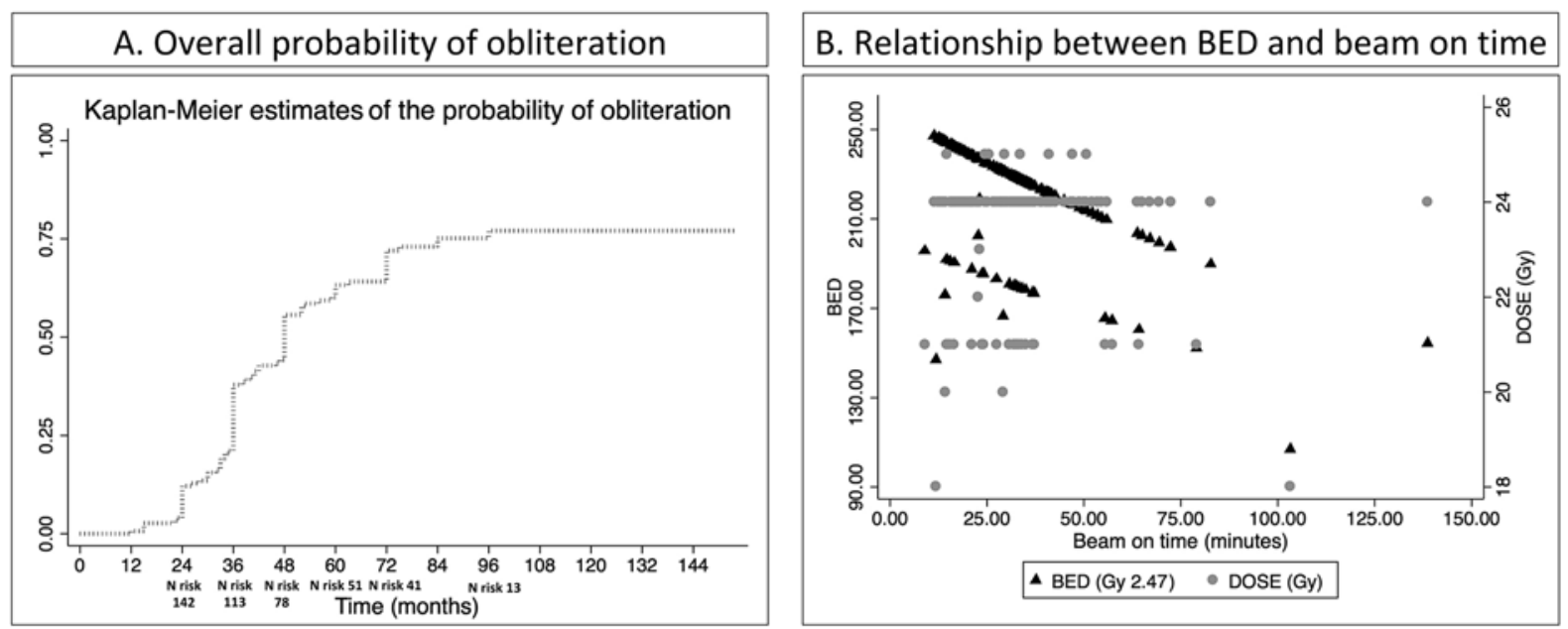

\section{BED as a positive predictor for obliteration}
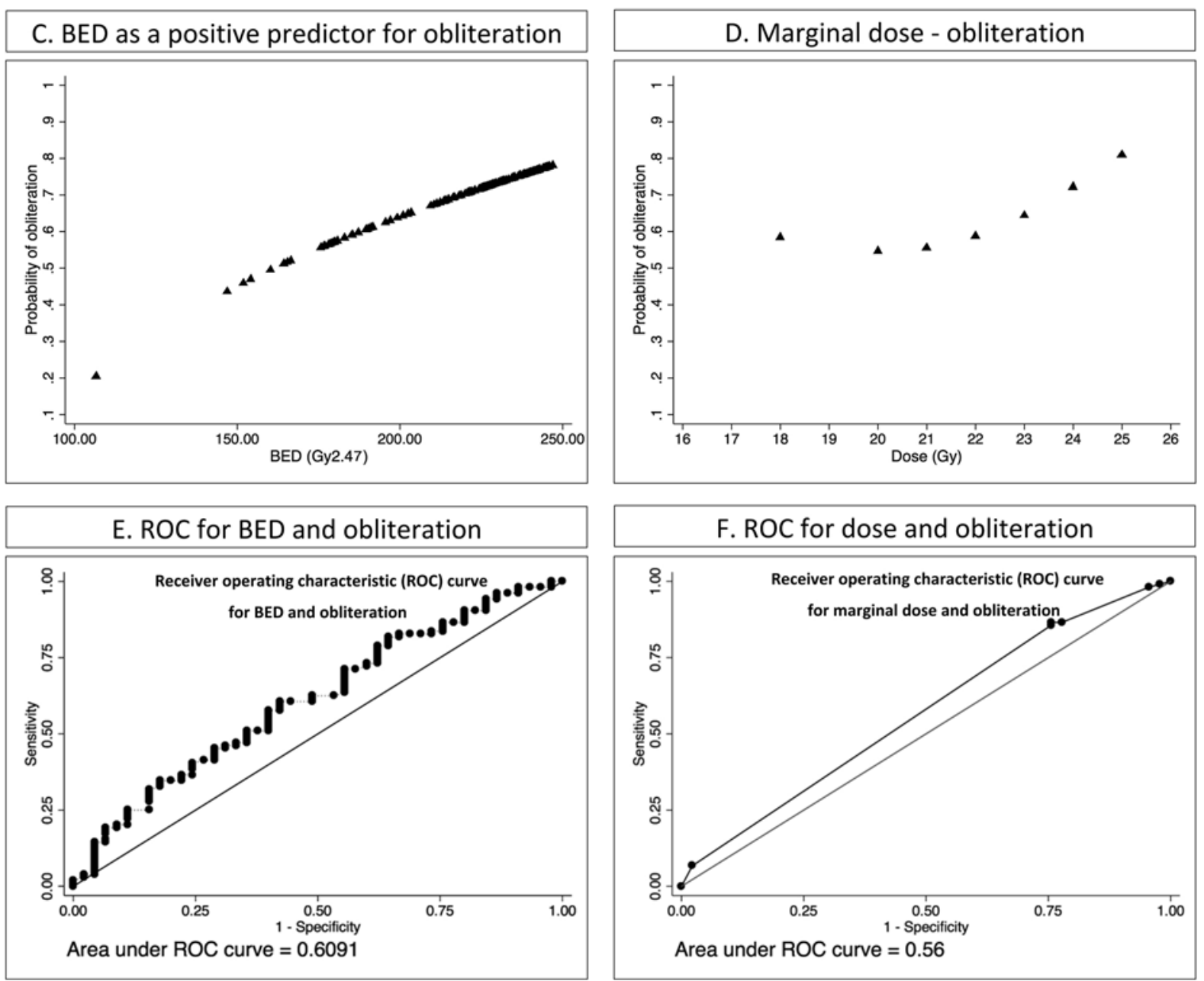

FIG. 1. Obliteration, with overall probability of obliteration (A), relationship between BED and beam-on time, showing that the vast majority of cases were treated with the same dose (24 Gy) (B), BED as a positive predictor for obliteration (C), marginal dose and obliteration (D), ROC for BED and obliteration (E), and ROC for dose and obliteration (F).

\section{Obliteration Score Using BED, Beam-On Time, V12, and Age (as a modulator)}

A radiosurgical obliteration score is derived from the fitted multivariable model as the sum of weighted factors according to their $\beta$-coefficients $(\beta=\log (\mathrm{OR}))$ : score $=$ $(0.018 \times \mathrm{BED})+(1.58 \times \mathrm{V} 12)+(-0.013689 \times$ beam-on time $)+(0.021 \times$ age $)-4.38$ (Fig. $2 \mathrm{~A}$ and $\mathrm{B})$. The score ranges from -2.78 to 2.62 (Fig. $2 \mathrm{C}$ ). A higher score cor- 

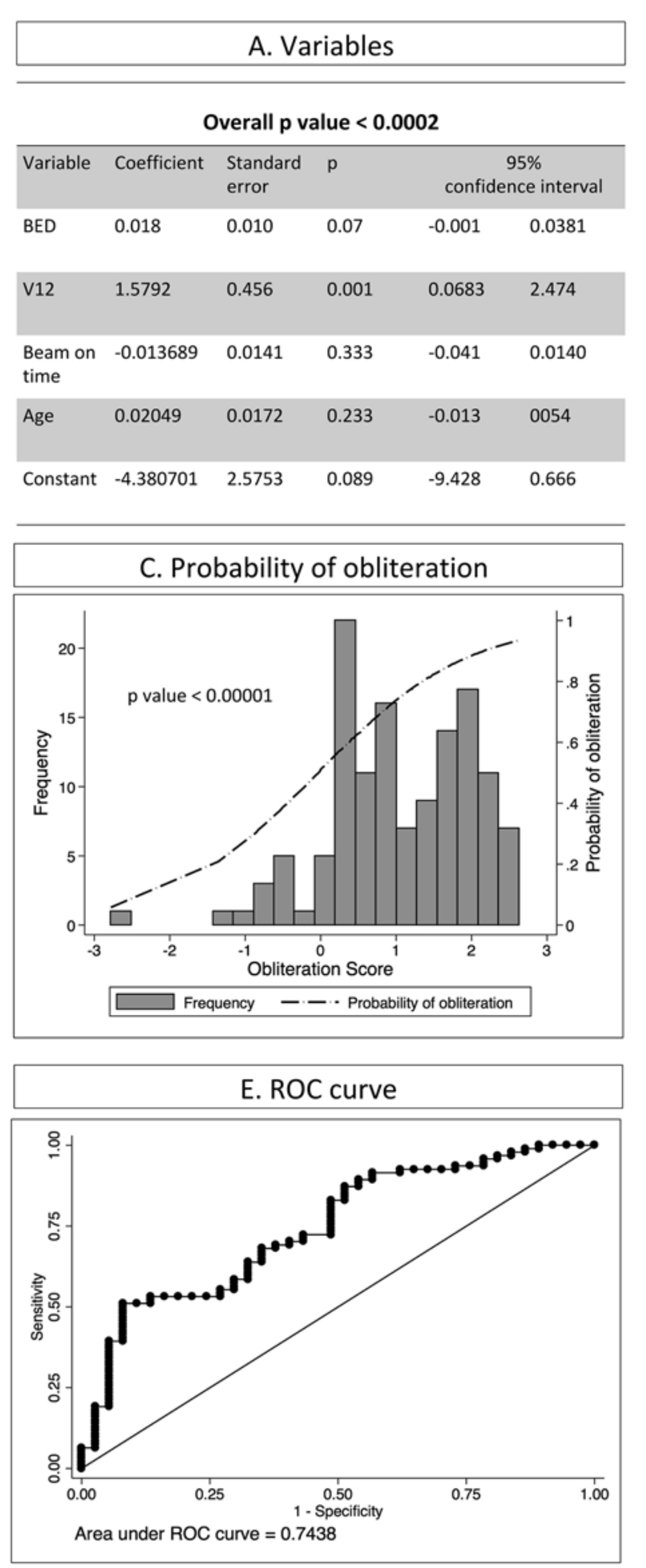

responds to higher probability of obliteration (Fig. 2C; the adjusted predictions with 95\% CI are illustrated in Fig. 2D). The score positively predicted the obliteration ( $\mathrm{p}<$ 0.00001; OR 2.72 [SE 0.68], 95\% CI 1.664-4.444). The AUC was 0.7438 (Fig. 2E).
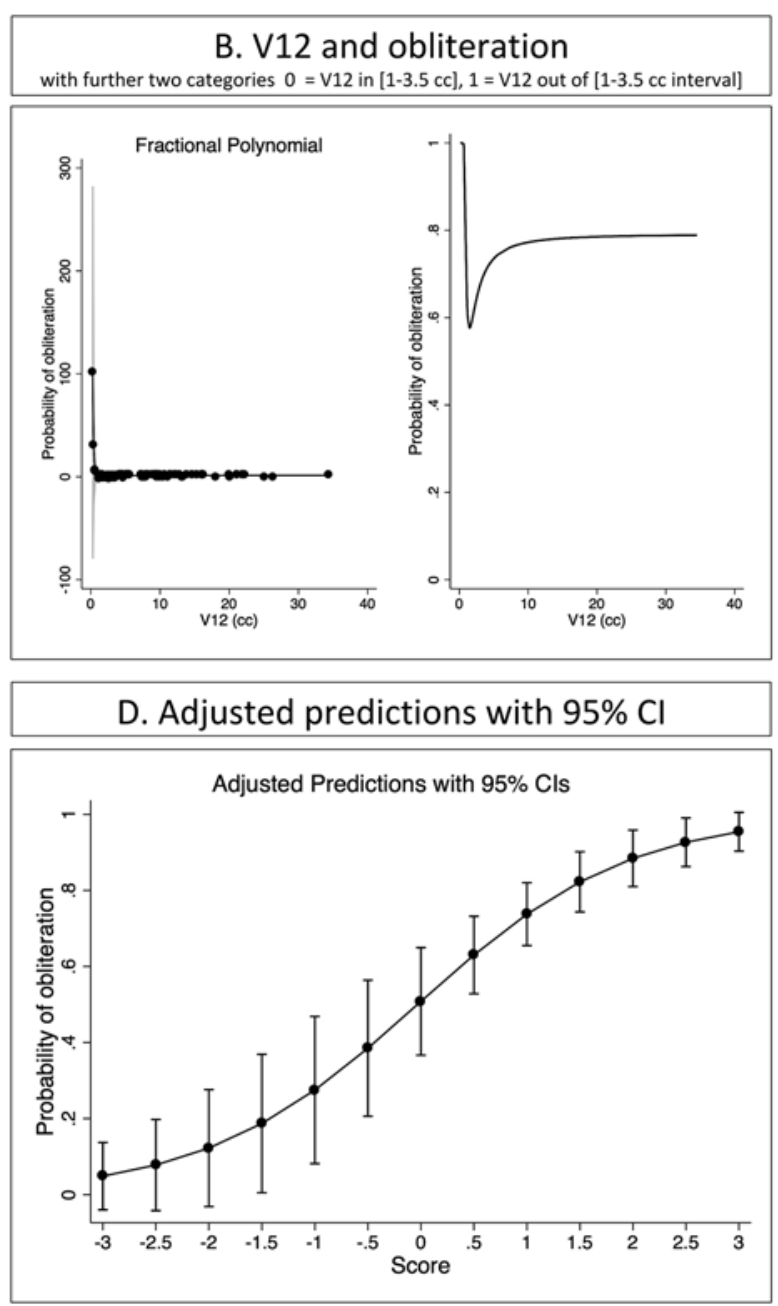

FIG. 2. Obliteration score, with variables of the score and the respective coefficients, standard errors, $p$ values, and $95 \%$ confidence intervals (A); V12 and obliteration and the further V12 categories within the statistical multivariate analysis (B); probability of obliteration using the obliteration score (C); adjusted predictions with 95\% confidence intervals (D); and ROC curve (E).

\section{Internal Validation of the Score Using the Bootstrap Method}

Internal validation of the score using the bootstrap method estimates the optimism at $3.5 \%$. Knowing that the AUC (original model) is 0.7438 , one would expect that 


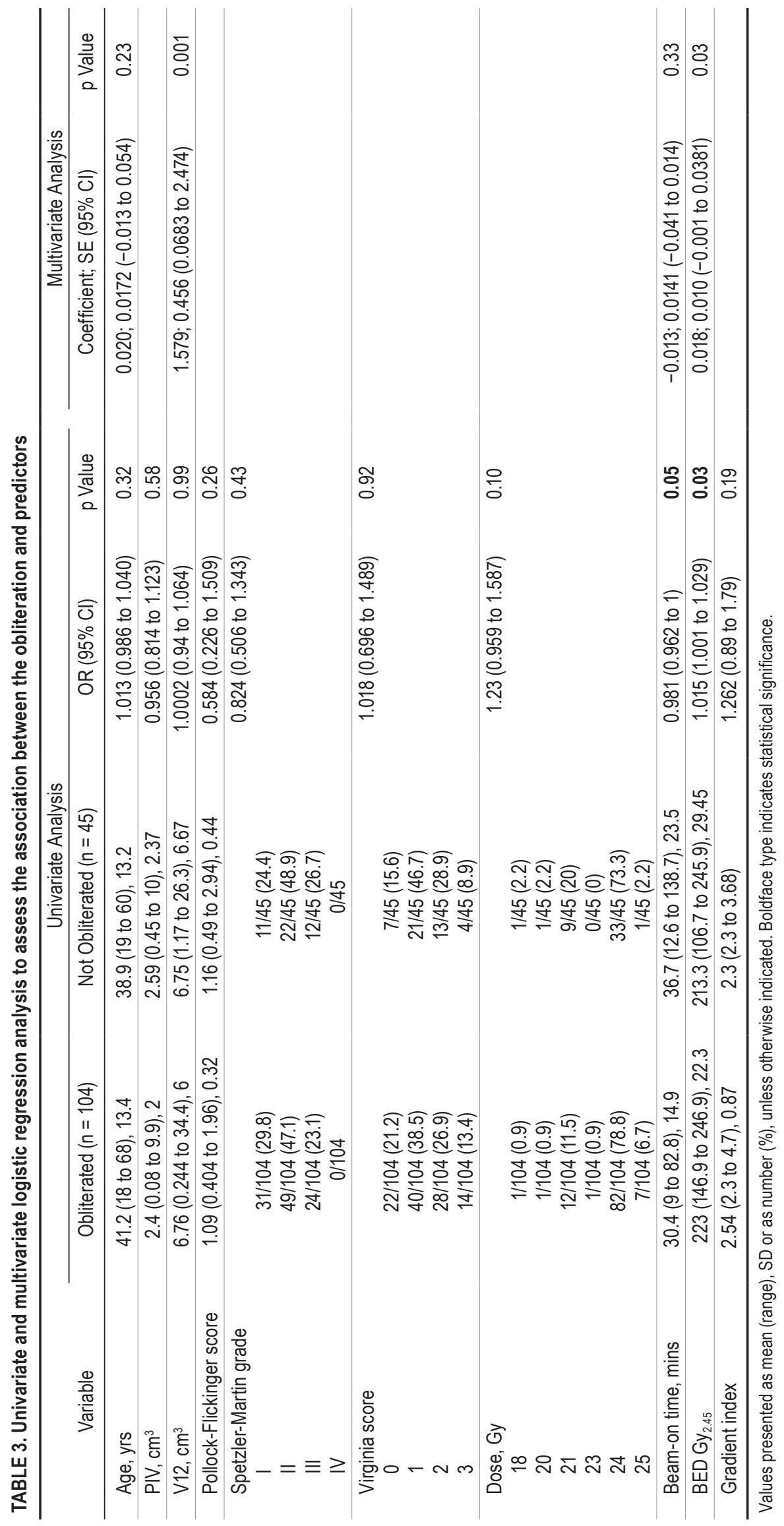


TABLE 4. Univariate logistic regression analysis to assess the association between the occurrence of complications and related predictors

\begin{tabular}{lll}
\hline \multicolumn{1}{c}{ Variable } & \multicolumn{1}{c}{ OR $(95 \% \mathrm{Cl})$} & p Value \\
\hline Age, yrs & $1.002(0.969-1.036)$ & 0.9 \\
\hline PIV $\mathrm{cm}^{3}$ & $1.3(1.081-1.562)$ & $\mathbf{0 . 0 0 5}$ \\
\hline V12, $\mathrm{cm}^{3}$ & $1.120(1.043-1.202)$ & $\mathbf{0 . 0 0 1}$ \\
\hline Pollock-Flickinger score & $4.637(1.382-15.558)$ & $\mathbf{0 . 0 2}$ \\
\hline Spetzler-Martin grade & $1.856(0.976-3.526)$ & 0.06 \\
\hline Virginia score & $2.180(1.304-3.644)$ & $\mathbf{0 . 0 0 3}$ \\
\hline Dose, Gy & $1.017(0.722-1.432)$ & 0.9 \\
\hline Beam-on time, mins & $0.966(0.931-1.002)$ & $\mathbf{0 . 0 3}$ \\
\hline BED Gy ${ }_{2.45}$ & $1.010(0.989-1.031)$ & 0.94 \\
\hline Gradient index & $0.773(0.511-1.168)$ & 0.22 \\
\hline
\end{tabular}

The sample size of patients developing complications, 20/149, precluded a multivariate analysis. Boldface type indicates statistical significance.

the power of discrimination of the score between patients with and without obliteration in an external cohort will be $74.38 \%-3.5 \%=70.88 \%$.

\section{Adverse Radiation Effects}

Twenty-eight (18.8\%) patients developed complications after GKRS. Transient radiological complications were encountered in $20(13.4 \%)$ patients, in whom transient perilesional edema developed. Three (2\%) patients developed asymptomatic hemorrhage after GKRS. Definitive clinical complications were present in $5(3.4 \%)$ patients: $3(2 \%)$ cases of motor hemiparesis, $1(0.7 \%)$ case of sensory hemiparesis, and $1(0.7 \%)$ case of intractable epilepsy.

Predictors for complication appearance after univariate analysis (Table 4) were higher PIV ( $\mathrm{p}=0.005), \mathrm{V} 12(\mathrm{p}=$ $0.001)$, and Pollock-Flickinger $(p=0.02)$ and Virginia $(p=$ $0.003)$ scores and lower beam-on time $(p=0.03)$.

\section{Discussion}

\section{BED, the Strongest Predictor for Unruptured AVM Obliteration After Upfront GKRS}

The management of unruptured AVMs remains controversial. Here, we evaluated the role of SRS in a population of patients treated before the appearance of the ARUBA trial (A Randomized Trial of Unruptured Brain Arteriovenous Malformations)..$^{28}$ Moreover, our purpose was to focus on the BED's role in relationship to the clinical and radiological outcomes. We report actuarial probabilities of obliteration at $36,60,72$, and 96 months of $37.9 \%, 63.3 \%$, $72 \%$, and $76 \%$, respectively. The BED was the strongest predictor of obliteration. In a multivariate model, the interaction between obliteration and BED, V12, beam-on time, and age (as a modulator) was positively correlated with higher obliteration rates. No statistically significant relationship between RDR and obliteration was found ( $\mathrm{p}=$ 0.29). Interestingly, the AUC for RDR was 0.56, identical to the one found for dose and obliteration. A radiosurgical score was proposed on the basis of these variables, with an AUC of 0.7438 . Twenty-eight (18.8\%) patients developed adverse radiation effects after GKRS, of which a vast majority were radiological and transient.

\section{Evaluating the Role of BED in General}

Initially, the impact of the RDR was evaluated for functional disorders, mainly trigeminal neuralgia, with heterogeneous results. ${ }^{29,30}$ The RDR designates the rate of radiation dose delivery, described as the amount of radiation absorbed by tissue per unit time. This was based on the evidence that ${ }^{60} \mathrm{Co}$ radioactivity has a half-life of 5.26 years and thus might have an impact on the radiobiological effect. However, these aspects have been recently questioned by several authors, suggesting treatment time as more relevant. ${ }^{31,32}$ In multi-isocentric GKRS planning, treatment time might vary by a factor of 10 , as previously stated..$^{32}$ It has been recognized for many decades that the biological effectiveness of a given radiation dose declines as a function of increasing exposure time. ${ }^{33} \mathrm{~A}$ parameter incorporating both the radiation dose and treatment time would be necessary. This parameter is currently considered to be the BED. However, its real effects in GKRS planning remain largely undiscovered. Of note, this methodology was initially suggested by Millar and Canney in $1993^{34}$ and further applied to GKRS by Hopewell et al. ${ }^{35}$ The difficulty in applying such a concept comes from the fact that its calculation in multi-isocentric plans is not simple. Here, we used a monoexponential fit to evaluate the individual BED values, using the unique combination of marginal dose and time. In sum, BED has a direct relationship to time, while RDR does not. In fact, in the present study, the beam-on time (i.e., not treatment time) was also statistically significant for obliteration, as a second strong parameter after BED. However, the RDR was not significantly associated with obliteration. Moreover, beam-on time was also significant for complication appearance after univariate analysis (the complications sample size precluded multivariate analysis). The exact threshold for safety and efficacy remains to be established by further studies.

\section{Evaluating the BED Role in Unruptured AVMs}

For radiobiological reasons, we aimed at studying the BED's role in nontumoral and nonfunctional neurosurgical pathology. Indeed, the mechanism of action of singlefraction SRS and GKRS in particular differs according to the condition being treated. These effects are mainly dose, volume, and time dependent. In Leksell's view, GKRS was initially conceived to create a focused necrotic lesion in functional indications. ${ }^{36}$ This had further evolved to destruction of pathological tissue (e.g., pituitary adenomas and vestibular schwannomas) or AVM obliteration by proliferative vasculopathy. ${ }^{17}$ Here, we focused on this former radiobiological effect. The study of BED was particularly of interest in unruptured AVMs, which underwent upfront GKRS and were obliterated without the need for further therapies. This allowed us to eliminate potential bias, including, for example, those related to embolic agent presence and staged GKRS treatments for larger volumes, with a potentially different radiobiological effect.

One could also question the role of such an approach involving a concept that is mathematically challenging and 
which has been just recently applied to SRS. The link between dose and BED can be also discussed. The key core remains, in our opinion, the time factor. Of note, in the present analysis, a vast majority of patients were treated with $24 \mathrm{~Gy}$ at the margin. This homogeneity in the prescribed dose, unlike the heterogeneity potentially given by including historical cases, most probably explains dose insignificance in the univariate analysis. Some authors have suggested that the percent volume of an AVM that receives a minimum dose of 20-23 Gy may be the more clinically relevant guide to obliteration. ${ }^{37}$ In fact, the Pittsburgh group's data might suggest adding additional isocenters so that $\geq 63 \%$ of the internal AVM dose receives more than $20 \mathrm{~Gy}^{37}$

\section{The BED Radiosurgery Obliteration Score}

In the present analysis, the proposed BED radiosurgery obliteration score includes the BED, beam-on time, and age. However, it also includes V12. Classically, V12 has been seen as a predictor for toxicity. ${ }^{38}$ Nevertheless, recent studies from the Charlottesville group suggested that unruptured AVM obliteration after SRS is also significantly associated with the radiological presence of radiationinduced changes (defined, as in the present study, as T2 hyperintensity surrounding the treated AVM nidus on MRI) during follow-up. ${ }^{39}$ This opens the question of a potential therapeutic window between efficacy and toxicity, as linked to changes within the adjacent brain parenchyma after SRS. In this respect, although positive predictors for AVM obliteration have been classically considered higher radiation dose and smaller treatment volumes, ${ }^{40}$ some other factors might be involved, including (but not limited to) V12. An internal validation of the obliteration score by bootstrap was performed.

An open question remains the exact BED therapeutic window between efficacy and toxicity for the particular case of GKRS for AVMs. The low number of persistent complications in the present analysis precluded such analysis.

\section{Limitations}

Our study has several inherent limitations. The first is related to the retrospective nature of the present analysis, with potential multiple biases. The second is the use of a monoexponential fit for BED calculation, thus a simplified formula. However, we wanted to provide the reader with a simple, yet clinically relevant, analysis with a potential translational impact for daily practice. The third is that our findings and the proposed score might be applied only to unruptured AVMs. The fourth is related to a limited sample size. Ideally, these findings should be replicated in larger cohorts. The same would apply to the proposed score, with a validation in both other centers and larger series. Another limitation is related to the absence from this cohort of Spetzler-Martin grades IV and V, especially. However, these patients present with larger AVM volumes, benefitting from previous embolization and/or volumestaged GKRS. These cases are reputed to have different results in terms of obliteration and, most probably, radiobiological effects. As stated in the Methods, they have been excluded from the present analysis, as our purpose was to analyze unruptured AVMs treated with upfront GKRS. In this respect, our results could be further applied only to similar cases, as described here. Another limitation is related to the fact that, in $80 \%$ of the cases in the present cohort, DSA confirmed the obliteration, while MRI confirmed the remaining $20 \%$. This could potentially have further overestimated the obliteration rates; however, this might have been the case in a small proportion of patients. Moreover, during their long-term follow-up, no particular recanalization was noted while performing follow-up MRI. Another limitation is that the exact time point of obliteration might be difficult to establish in some patients. This is mainly due to the fact that, as a French referral center for AVM radiosurgical treatment, we treat patients from a large territory. Some of the patients come on the exact date and others with various delays in their followup time points. However, we preferred to take into account in our analysis the exact date of the follow-up point for all cases. This might also have engendered an underestimation of the obliteration rates at 3 years.

\section{Perspectives}

Radiosurgery would potentially evolve toward a planning system incorporating a desired radiobiological effect. In this respect, BED would play a key role.

\section{Conclusions}

Our results confirm that upfront GKRS for unruptured AVMs treated before the ARUBA trial is safe and effective. The BED was the strongest predictor of obliteration, followed by treatment time. A radiosurgical score of obliteration on the basis of BED is proposed. An internal validation of this score was performed using the bootstrap method. Treatment time was illustrated as statistically significant for both obliteration and complication appearance. The exact threshold remains to be established by further studies. Special interest should be given to prospective trials to validate such an approach.

\section{Acknowledgments}

We acknowledge Lille University Hospital, Lausanne University Hospital (CHUV), and University of Lausanne.

Constantin Tuleasca gratefully acknowledges the receipt of a Young Researcher in Clinical Research Grant (Jeune Chercheur en Recherche Clinique) from the University of Lausanne, Faculty of Biology and Medicine, and the Lausanne University Hospital (CHUV).

\section{References}

1. Friedlander RM. Clinical practice. Arteriovenous malformations of the brain. N Engl J Med. 2007;356(26):2704-2712.

2. Solomon RA, Connolly ES Jr. Arteriovenous malformations of the brain. N Engl J Med.2017;376(19):1859-1866.

3. Spetzler RF, Hargraves RW, McCormick PW, et al. Relationship of perfusion pressure and size to risk of hemorrhage from arteriovenous malformations. J Neurosurg. 1992;76(6): 918-923.

4. Hernesniemi JA, Dashti R, Juvela S, et al. Natural history of brain arteriovenous malformations: a long-term follow-up study of risk of hemorrhage in 238 patients. Neurosurgery. 2008;63(5):823-831. 
5. Al-Shahi R, Bhattacharya JJ, Currie DG, et al. Prospective, population-based detection of intracranial vascular malformations in adults: the Scottish Intracranial Vascular Malformation Study (SIVMS). Stroke. 2003;34(5):1163-1169.

6. Lawton MT. Spetzler-Martin Grade III arteriovenous malformations: surgical results and a modification of the grading scale. Neurosurgery. 2003;52(4):740-749.

7. Morgan MK, Rochford AM, Tsahtsarlis A, et al. Surgical risks associated with the management of Grade I and II brain arteriovenous malformations. Neurosurgery. 2007;61(1) (suppl):417-424.

8. Spetzler RF, Martin NA. A proposed grading system for arteriovenous malformations. J Neurosurg. 1986;65(4):476-483.

9. Hartmann A, Pile-Spellman J, Stapf C, et al. Risk of endovascular treatment of brain arteriovenous malformations. Stroke. 2002;33(7):1816-1820.

10. Graffeo CS, Sahgal A, De Salles A, et al. Stereotactic radiosurgery for Spetzler-Martin grade I and II arteriovenous malformations: International Society of Stereotactic Radiosurgery (ISRS) practice guideline. Neurosurgery. Published online February 17, 2020. doi:10.1093/neuros/nyaa004

11. Pollock BE, Flickinger JC, Lunsford LD, et al. Hemorrhage risk after stereotactic radiosurgery of cerebral arteriovenous malformations. Neurosurgery. 1996;38(4):652-661.

12. Mathis JA, Barr JD, Horton JA, et al. The efficacy of particulate embolization combined with stereotactic radiosurgery for treatment of large arteriovenous malformations of the brain. AJNR Am J Neuroradiol. 1995;16(2):299-306.

13. Rubin BA, Brunswick A, Riina H, Kondziolka D. Advances in radiosurgery for arteriovenous malformations of the brain. Neurosurgery. 2014;74(suppl 1):S50-S59.

14. Sirin S, Kondziolka D, Niranjan A, et al. Prospective staged volume radiosurgery for large arteriovenous malformations: indications and outcomes in otherwise untreatable patients. Neurosurgery. 2008;62(suppl 2):744-754.

15. Millar WT, Hopewell JW, Paddick I, et al. The role of the concept of biologically effective dose (BED) in treatment planning in radiosurgery. Phys Med. 2015;31(6):627-633.

16. Tuleasca C, Paddick I, Hopewell JW, et al. Establishment of a therapeutic ratio for Gamma Knife radiosurgery of trigeminal neuralgia: the critical importance of biologically effective dose versus physical dose. World Neurosurg. 2020;134:e204e213.

17. Szeifert GT, Timperley WR, Forster DMC, Kemeny AA. Histopathological changes in cerebral arteriovenous malformations following Gamma Knife radiosurgery. Prog Neurol Surg. 2007;20:212-219.

18. Kano H, Kondziolka D, Flickinger JC, et al. Aneurysms increase the risk of rebleeding after stereotactic radiosurgery for hemorrhagic arteriovenous malformations. Stroke. 2012; 43(10):2586-2591.

19. Schwyzer L, Yen CP, Evans A, et al. Long-term results of gamma knife surgery for partially embolized arteriovenous malformations. Neurosurgery. 2012;71(6):1139-1148.

20. Reyns N, Blond S, Gauvrit JY, et al. Role of radiosurgery in the management of cerebral arteriovenous malformations in the pediatric age group: data from a 100-patient series. Neurosurgery. 2007;60(2):268-276.

21. Jones B, Hopewell JW. Modelling the influence of treatment time on the biological effectiveness of single radiosurgery treatments: derivation of "protective" dose modification factors. Br J Radiol. 2019;92(1093):20180111.

22. Lunsford LD, Kondziolka D, Flickinger JC, et al. Stereotactic radiosurgery for arteriovenous malformations of the brain. $J$ Neurosurg. 1991;75(4):512-524.

23. Flickinger JC, Kondziolka D, Maitz AH, Lunsford LD. Analysis of neurological sequelae from radiosurgery of arteriovenous malformations: how location affects outcome. Int $J$ Radiat Oncol Biol Phys. 1998;40(2):273-278.
24. Starke RM, Yen CP, Ding D, Sheehan JP. A practical grading scale for predicting outcome after radiosurgery for arteriovenous malformations: analysis of 1012 treated patients. $J$ Neurosurg. 2013;119(4):981-987.

25. Pollock BE, Flickinger JC. A proposed radiosurgery-based grading system for arteriovenous malformations. J Neurosurg. 2002;96(1):79-85.

26. Leclerc X, Guillaud O, Reyns N, et al. Follow-up MRI for small brain AVMs treated by radiosurgery: is gadolinium really necessary? AJNR Am J Neuroradiol. 2020;41(3):437_ 445.

27. Harrell FE Jr, Lee KL, Mark DB. Multivariable prognostic models: issues in developing models, evaluating assumptions and adequacy, and measuring and reducing errors. Stat Med. 1996;15(4):361-387.

28. Mohr JP, Parides MK, Stapf C, et al. Medical management with or without interventional therapy for unruptured brain arteriovenous malformations (ARUBA): a multicentre, nonblinded, randomised trial. Lancet. 2014;383(9917):614-621.

29. Arai Y, Kano H, Lunsford LD, et al. Does the Gamma Knife dose rate affect outcomes in radiosurgery for trigeminal neuralgia? J Neurosurg. 2010;113(suppl):168-171.

30. Lee JY, Sandhu S, Miller D, et al. Higher dose rate Gamma Knife radiosurgery may provide earlier and longer-lasting pain relief for patients with trigeminal neuralgia. $J$ Neurosurg. 2015;123(4):961-968.

31. Paddick I, Hopewell JW, Klinge T, et al. Treatment outcomes and dose rate effects following Gamma Knife stereotactic radiosurgery for vestibular schwannomas. Letter. Neurosurgery. 2020;86(4):E407-E409.

32. Tuleasca C, Régis J, Levivier M. Treatment outcomes and dose rate effects following Gamma Knife stereotactic radiosurgery for vestibular schwannomas. Letter. Neurosurgery. 2020;86(2):E252-E253.

33. Canney PA, Millar WT. Biphasic cellular repair and implications for multiple field radiotherapy treatments. Br J Radiol. 1997;70(836):817-822.

34. Millar WT, Canney PA. Derivation and application of equations describing the effects of fractionated protracted irradiation, based on multiple and incomplete repair processes. Part I. Derivation of equations. Int J Radiat Biol. 1993;64(3): 275-291.

35. Hopewell JW, Millar WT, Lindquist C. Radiobiological principles: their application to $\gamma$ knife therapy. Prog Neurol Surg. 2012;25:39-54.

36. Leksell L. Cerebral radiosurgery. I. Gammathalanotomy in two cases of intractable pain. Acta Chir Scand. 1968;134(8): $585-595$.

37. Kano H, Flickinger JC, Nakamura A, et al. How to improve obliteration rates during volume-staged stereotactic radiosurgery for large arteriovenous malformations. J Neurosurg. 2019;130(6):1809-1816.

38. Flickinger JC, Kondziolka D, Lunsford LD, et al. Development of a model to predict permanent symptomatic postradiosurgery injury for arteriovenous malformation patients. Int J Radiat Oncol Biol Phys. 2000;46(5):1143-1148.

39. Ding D, Yen CP, Xu Z, et al. Radiosurgery for patients with unruptured intracranial arteriovenous malformations. J Neurosurg. 2013;118(5):958-966.

40. Liscák R, Vladyka V, Simonová G, et al. Arteriovenous malformations after Leksell gamma knife radiosurgery: rate of obliteration and complications. Neurosurgery. 2007;60(6): 1005-1016.

41. Graffeo CS, Donegan D, Erickson D, et al. The impact of insulin-like growth factor index and biologically effective dose on outcomes after stereotactic radiosurgery for acromegaly: cohort study. Neurosurgery. Published online April 8, 2020. doi:10.1093/neuros/nyaa054

42. Régis J, Tuleasca C, Hopewell JW, Castinetti F. Commentary: 
The impact of insulin-like growth factor index and biological effective dose on outcomes after stereotactic radiosurgery for acromegaly: cohort study. Neurosurgery. Published online April 29, 2020. doi:10.1093/neuros/nyaa123nyaa123

\section{Disclosures}

Dr. Tuleasca: scientific advisor for Elekta AB.

\section{Author Contributions}

Conception and design: Tuleasca, Reyns. Acquisition of data: Peciu-Florianu, Leroy, Tuleasca (dose rate, BED). Analysis and interpretation of data: Tuleasca, Reyns. Drafting the article:
Tuleasca. Critically revising the article: all authors. Reviewed submitted version of manuscript: all authors. Approved the final version of the manuscript on behalf of all authors: Tuleasca. Statistical analysis: Tuleasca, Faouzi. Administrative/technical/ material support: Tuleasca, Faouzi, Reyns. Study supervision: Tuleasca, Reyns.

\section{Correspondence}

Constantin Tuleasca: University of Lausanne, Faculty of Biology and Medicine and Centre Hospitalier Universitaire Vaudois (CHUV), Lausanne, Switzerland. constantin.tuleasca@chuv.ch; constantin.tuleasca@gmail.com. 